\title{
Pilotless Carrier Phase-Synchronization via LDPC Code Feedback
}

\author{
Esteban L. Vallés, Richard D. Wesel, and John D. Villasenor \\ University of California, Los Angeles. \\ Electrical Engineering Department. \\ $\{$ evalles, wesel, villa\}@ee.ucla.edu.
}

\author{
Christopher R. Jones, and Marvin Simon \\ Jet Propulsion Laboratory \\ $\{$ christop, msimon\}@jpl.nasa.gov.
}

\begin{abstract}
This paper addresses the carrier-phase estimation problem under the low SNR conditions often encountered in turbo and LDPC-coded applications. In [1] a decision-directed carrier synchronization (DDCS) circuit that uses soft information from an iterative LDPC decoder was presented for BPSK and QPSK constellations with constant phase offsets. In this work, we present a method that is able to handle arbitrary constellations with a random-walk phase noise process. Loop SNR equations are derived and the performance for different constellations is shown. An extension of the DDCS algorithm is described that uses a search and tracking method based on measuring the number of satisfied LDPC constraint-node equations equations at the decoder and is able to track arbitrary carrier phase.
\end{abstract}

\section{INTRODUCTION}

In recent years there has been increasing interest in highly efficient error-correction codes such as turbo codes and low density parity check (LDPC) codes. These codes operate at very low symbol signal-to-noise ratios (SNRs) thus requiring carrier synchronization schemes that can track the carrier at these SNRs.

A significant research effort is underway in the area of joint decoding and carrier phase estimation. As described by Noels et al. [2], two somewhat distinct groups of joint decoding and synchronization algorithms have evolved. The first of these groups, approaches the problem by modifying iterative detection/decoding algorithms and/or graphs to include parameter estimation. A partial list of such work includes [3]-[5]. Of particular interest has been the work of Colavolpe et al. [5] where phase-tracking processing nodes were introduced in the iterative decoding graph. Dauwels et al. [4] also investigated specially adapted message-passing update rules. Howard et al. [6] proposed a pilotless modulation technique for turbocoded differential 8PSK modulation which uses 35 iterations to compensate a $\pi / 8$ phase offset at $E_{b} / N_{o}=4.5 \mathrm{~dB}$. We also note the work of Nuriyev and Anasatasopoulos [7] on adapting density evolution to evaluate the performance of joint carrierphase estimation in a pilot-assisted environment. The second group of algorithms passes messages between an independent phase estimation block and an essentially unmodified iterative decoder. The resulting architectures are often said to employ turbo synchronization [8]. Algorithms of this type can can be found in [8]-[13].

The technique in this paper falls into this second category and has the potentially attractive feature that little modification is required with either the iterative decoder or the carrier recovery block (which consists primarily of a phase-locked loop (PLL)). Specifically, the work leverages the fact that LDPC symbol estimates can 'wipe-off' modulated symbols in a decision-directed carrier recovery loop, to enhance the carrier information such that a classic residual carrier PLL is able to provide increasingly accurate phase estimates over LDPC iterations. The method incurs a latency penalty (by way of increased iterations) as carrier phase is acquired. However, complexity in terms of system description and area (in the case of a real-time implementation) remains similar to that of state of the art residual carrier recovery techniques currently used, for example, in NASA's deep-space network. Moreover, the proposed architecture can be used in conjunction with other types of phase tracking loops in order to track residual carrierphase errors at the decoding stage. We also note that if pilot information is available, it can be incorporated to the proposed carrier recovery loop at no additional cost.

The authors in [11] have developed a blind recovery technique for QAM receivers. The work in this paper is also based on blind, or pilotless, operation and we motivate this in part by recalling a result from Anastasopoulos [3] who showed pilotless techniques to be more efficient at lower SNRs where pilot insertion loss is considerable.

The rest of this paper is organized as follows. The next section provides a detailed description of the decision-directed carrier synchronization (DDCS) method. We derive the tracking performance of a PLL in terms of its mean-square phase error when operating in the linear (high loop-SNR) region as is typical. In Section III we explain the iterative phase tracking algorithm. Results for different modulations are presented in Section IV. Finally, Section V gives conclusions.

\section{TRACKING PERFORMANCE}

On the transmitter side, we consider a baseband signal comprised of root raised-cosine pulses $p(t)$, transmitted at multiples of a symbol interval $T_{s}: M(t)=m_{I}(t)+j m_{Q}(t)=$ $\sum_{k=-\infty}^{\infty} d_{I_{k}} p\left(t-k T_{s}\right)+j \sum_{k=-\infty}^{\infty} d_{Q_{k}} p\left(t-k T_{s}\right)$. Multiplication by a sinusoidal carrier signal yields the transmitted waveform:

$$
Y_{T}(t)=M(t) e^{j\left(\omega_{c} t\right)}=y_{T_{I}}(t)+j y_{T_{Q}}(t)
$$


where $\omega_{c}$ is the carrier frequency. A constant envelope modulation with $\left\{d_{I_{k}}, d_{Q_{k}}\right\} \in\{ \pm \sqrt{P / 2}\}$ ( $P$ is the carrier power $)$ is initially assumed, although the proposed carrier recovery method works for any type of constellation as described in Section IV. In the transmission process, the signal in (1) is rotated by an angle $\theta_{c}$ and affected by a bandpass AWGN process, $N(t)=\left(n_{I}(t)+j n_{Q}(t)\right) e^{j\left(\omega_{c} t+\theta_{c}\right)}$, where $n_{I}(t)$ and $n_{Q}(t)$ have single-sided noise power spectral density (PSD) equal to $N_{0}$. The time-changing model for $\theta_{c}$ is described later

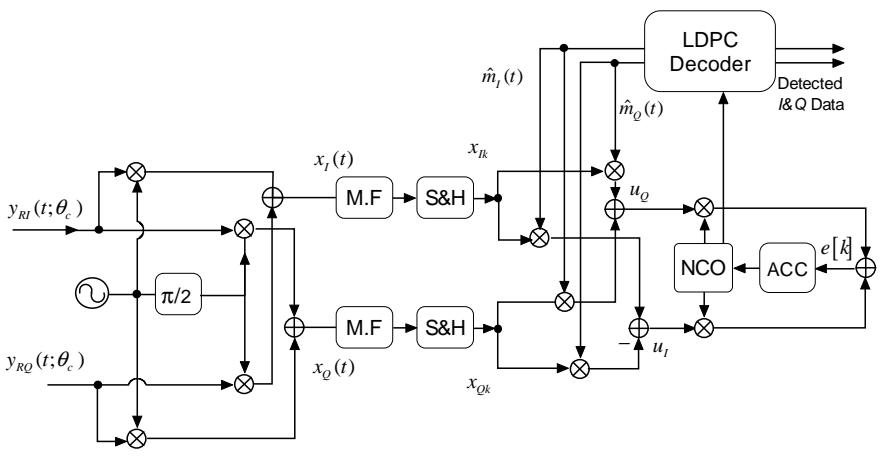

Fig. 1. Block diagram of an digital receiver

in this section. Accurately estimating $\theta_{c}$ is the goal of carrier synchronization. A sample receiver, shown in Fig. 1, will be referred to in deriving the proposed carrier synchronization method. At the receiver, consider an input modulation of the form:

$$
Y_{R}(t)=(M+N) e^{j\left(\omega_{c} t+\theta_{c}\right)}=y_{R_{I}}(t)+j y_{R_{Q}}(t),
$$

where the time reference $(t)$ has been dropped from the symbol vectors $M$ and $N$ (and their components) for ease of notation.

To illustrate the concept behind the DDCS algorithm, consider a very simple example where five BPSK symbols consisting of alternating 1's and -1's affected by symbol-wise noise (note that the amplitude of each pulse is different than unity) are transmitted as in Fig. 2(a). Modulation is removed by multiplying the received waveform by soft-estimated symbols from the LDPC decoder. The resulting unmodulated frequency spectrum, shown in Fig. 2(b), clearly begins to show the presence of a carrier. Note that this occurs regardless of the fact that some symbol estimates may still be incorrect. Since the information feedback consists of soft information, the incorrect data has in general low reliability. After the decoder has converged to the correct codeword, the magnitude of the tone in Fig. 2(b) will continue to increase, indicating that the PLL is successfully tracking the carrier.

Let us assume a constellation size $S$ and a mapping $\Psi(S)=$ $\vec{T}$ that associates every symbol $S$ with a binary vector $\vec{T}$ of size $\log _{2}(S)$. For every bit $i=1: \log _{2}(S)$ in a received symbol $Y_{R}$, the LDPC decoder computes the probability $\rho_{i}$ of each bit being zero or one. A complex estimate $\hat{M}$ can be derived using the following:

$$
\hat{M}=\sum_{j=1}^{S} M_{j} \prod_{i=1}^{\log _{2}(S)} \operatorname{Pr}\left(\rho_{i}=\vec{T}_{j i}\right) .
$$

In (3) we note that when the decoder only uses a-priori information then $\hat{M}=\overrightarrow{0}$. On the other hand when the likelihood of a particular symbol $j$ is very high then $\hat{M} \approx$ $M_{j}$. In this work, two different techniques for removing the modulation information present in the received waveform have been examined. The first proposed technique uses the complex conjugate (CC) of the estimated symbol, $\hat{M}^{*}(t)$, as feedback. The second proposed technique normalizes the symbol estimates to assure unit amplitude, $\hat{M}^{*}(t) /\left\|\hat{M}^{*}(t)\right\|$. The intuition behind these two choices is that when using CC feedback, both the magnitude and phase of the feedback vector are fed back to the carrier recovery circuit. On the other hand when the normalized complex conjugate (NCC) is used as a metric, only the phase of the estimated symbol is sent from the decoder to the DDCS circuit. The choice of the modulation removal technique depends on the signal constellation chosen for transmission and on the range of possible values that the carrier recovery circuit is intended to track. As will be described in Section IV, our experiments show that for constant envelope modulations both methods yield similar performance. For non-constant envelope modulations such as 16QAM and APSK, using the NCC as a metric is a better choice, especially for cases with large carrier offsets. There are many other possible alternatives that can be implemented by manipulating the LDPC output symbol estimates in different ways. This choice will depend on the modulation used and should be carefully designed jointly with the loop gains present in the PLL in order to minimize the frame error rate (FER).

\section{A. Complex Conjugate (CC) Feedback}

This technique uses the complex conjugate of the estimated symbol output from the LDPC decoder, $\hat{M}^{*}(t)$, to remove the modulation information present in (2):

$$
U(t)=Y_{R}(t) \hat{M}^{*}(t)=Y_{R}(t)\left(M^{*}(t)+N_{s}(t) / \eta\right)
$$

where $N_{s}=\sum_{k=-\infty}^{\infty}\left(n_{s_{I} k}+j n_{s_{Q} k}\right) p\left(t-k T_{s}\right)$, models the decoder symbol-estimation error and $\eta$ is the LDPC decoder a posteriori signal amplitude. Both $n_{s_{I} k}$ and $n_{s_{I} k}$ are i.i.d. $\mathcal{N}\left(0, \sigma_{n}{ }^{2}\right)$. By observing the symbol-estimation noise term $N_{s}(t) / \eta$ we can see how $\hat{M}(t) \rightarrow M(t)$ as the LDPC decoder iterations increase and the reliability of the a posteriori values increase. Let $u_{I}(t)=\Re\{U(t)\}, u_{Q}(t)=\Im\{U(t)\}$, then

$$
\begin{aligned}
& u_{I}(t)=(\underbrace{\left(m_{I} \hat{m}_{I}+m_{Q} \hat{m}_{Q}\right)}_{\alpha_{s}}+\underbrace{\left(n_{I} \hat{m}_{I}+n_{Q} \hat{m}_{Q}\right)}_{\alpha_{n}}) \cos \left(\omega_{c} t+\theta_{c}\right) \\
& +(\underbrace{\left(m_{I} \hat{m}_{Q}-m_{Q} \hat{m}_{I}\right)}_{\beta_{s}}+\underbrace{\left(n_{I} \hat{m}_{Q}-n_{Q} \hat{m}_{I}\right)}_{\beta_{n}}) \sin \left(\omega_{c} t+\theta_{c}\right) \\
& u_{Q}(t)=\left(\alpha_{s}+\alpha_{n}\right) \sin \left(\omega_{c} t+\theta_{c}\right)-\left(\beta_{s}+\beta_{n}\right) \cos \left(\omega_{c} t+\theta_{c}\right) .
\end{aligned}
$$


The signals above are then input to a PLL whose voltagecontrolled oscillator (VCO) output can be expressed as $r_{v c o}\left(t, \hat{\theta}_{c}\right)=\sin \left(\omega_{c} t+\hat{\theta}_{c}\right)$. Multiplying $u_{I}(t)$ and $u_{Q}(t)$ by $r_{v c o}\left(t, \hat{\theta}_{c}\right)$ and $r_{v c o}\left(t, \hat{\theta}_{c}-\pi / 2\right)=\cos \left(\omega_{c} t+\hat{\theta}_{c}\right)$ and combining the results of these products yields,

$$
\begin{aligned}
& z\left(t, \phi_{c}\right)=u_{Q}(t) \cos \left(\omega_{c} t+\hat{\theta}_{c}\right)-u_{I}(t) \sin \left(\omega_{c} t+\hat{\theta}_{c}\right) \\
& =\alpha_{s} \sin \left(\phi_{c}\right)+\underbrace{\left(\alpha_{n} \sin \left(\phi_{c}\right)-\left(\beta_{s}+\beta_{n}\right) \cos \left(\phi_{c}\right)\right)}_{v\left(t, \phi_{c}\right)}
\end{aligned}
$$

where $\phi_{c}=\theta_{c}-\hat{\theta}_{c}$. Note that no low-pass filter is required in (6) since the frequency components at $2 \omega_{c}$ have opposite signs and cancel each other.

Finally, analyzing the statistical properties of the loop's phase error as in [1], we arrive at the expression of the meansquare phase error in the loop,

$$
\sigma_{\phi_{c}}^{2}=\frac{N_{0} B_{L}}{P^{2}}\left(1+\left(\frac{1}{P}+\frac{1}{\sigma_{n}^{2}}\right) \frac{\sigma^{2}}{\eta^{2}}\right) \triangleq \frac{1}{\rho S_{L}},
$$

where $B_{L}$ is the noise bandwidth, $\rho=P /\left(N_{0} B_{L}\right)$ is the loopSNR in a conventional PLL and

$$
S_{L}^{D D C S} \triangleq\left(\frac{1}{P}+\left(\frac{1}{P^{2}}+\frac{1}{\sigma_{n}^{2}}\right) \frac{\sigma^{2}}{\eta^{2}}\right)^{-1},
$$

is the degradation of the loop-SNR analogous to the "squaring loss" in a conventional Costas loop (CL). The quantity $\eta^{2} / \sigma^{2}$ represents the decoder soft SNR estimate. As the iteration proceeds, the estimated data SNR increases and likewise the squaring loss decreases (i.e., $S_{L}^{D D C S} \rightarrow P$ ). By comparison, for a Costas loop, the expression for the squaring loss is given by

$$
S_{L}^{C} \triangleq\left(1+1 /\left(2 R_{d}\right)\right)^{-1}, R_{d}=P / N_{0}
$$

and thus remains fixed, independent of the iteration process, for a given symbol SNR [14].

To numerically evaluate the performance in (8), one needs to quantify the functional dependence of the decoder softestimate of the data SNR and the input symbol SNR. Under the assumptions in which (9) and (8) were derived, for the SNR regime where the DDCS circuit operates, the expected theoretical gain in $\mathrm{SL}$ at $\mathrm{SNR}=-1.5 \mathrm{~dB}$ is approximately $2.32 \mathrm{~dB}$. Both circuits were simulated at low SNR scenarios using the LDPC codes in the IEEE 802.11n standard. We note the simplicity of the resulting all-digital PLL-based-carrier phase recovery circuit in Fig. 1. The baseband portion of the proposed circuit requires 3 complex multiplies, 3 real additions, an numerically controlled oscillator (NCO) and an accumulator. Finally, this implementation, unlike that of a Costas loop, does not require a low pass filter to remove doubled frequency components.

Phase Noise Model: In order to model the carrier-phase noise process $\theta_{c_{k}}$, a random-walk (Wiener) model was studied,

$$
\theta_{c_{k}}=\theta_{c_{k-1}}+\Delta_{k} \text {. }
$$

This phase model implies a random-walk within a codeword, but is independent from one block to the next. After the DDCS

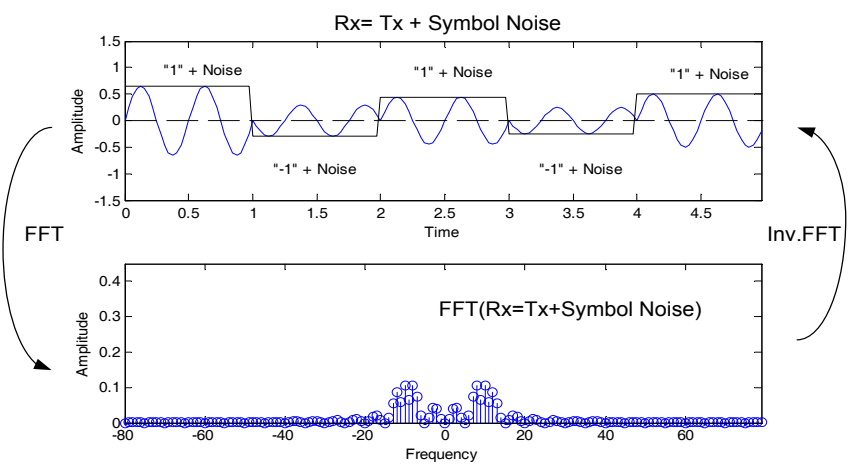

(a) Received Symbols $=$ Transmitted Symbols + Symbol-Noise

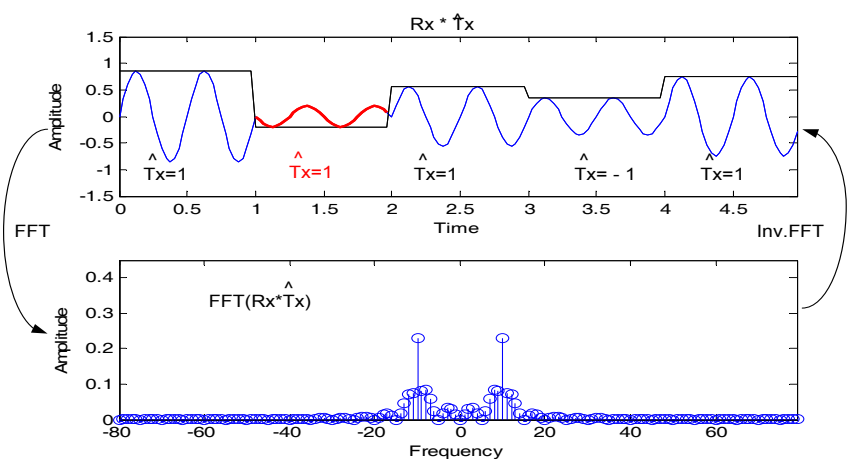

(b) DDCS-Demodulated Waveform

Fig. 2. DDCS BPSK Example. (a) Transmitted symbol sequence, $T_{x}=$ $[1 ;-1 ; 1 ;-1 ; 1]$ is shown with symbol-wise noise added, resulting in $R_{x}$. (b) After a small number of iterations, the imposed soft-estimates $\left(\hat{T}_{x}\right)$ from the DDCS loop are multiplied with $R_{x}$ to remove the modulation. Note that we have failed to remove the modulation on the second symbol (because the estimate of the symbol was incorrect), however a clear carrier signal which the PLL can track still emerges.

loop processes a block of $n$ symbols, the estimated carrier phase vector $\overrightarrow{\hat{\theta}}_{c}$, is used to de-rotate each of the symbols in the received channel observation vector. Performance using the carrier phase model in (10) together with a constant phase model are shown in Fig. 3. The different curves show the performance for different variances of Gaussian distributed $\left(\mathcal{N}\left(0, \sigma^{2}\right)\right)$ realizations of $\Delta_{k}$. The initial phase value, $\theta_{c_{0}}$, for each block, is drawn uniformly over the interval $[-\pi / 4, \pi / 4]$. Note that the pull-in range of the carrier recovery circuit ultimately spans the unit circle $([-\pi, \pi])$, but the nature of the joint phase estimation/decoding process requires that we resolve phase ambiguity in order to ensure successful decoding. Section III explains methods we have used to resolve constellation phase ambiguities. Note, however, that in much of what follows we endeavor to describe the pull-in performance of the proposed carrier recovery technique up to the limit of the rotational invariance angle for each examined constellation. Results show a loop-SNR gain in steady state for the DDCS circuit of $1.5 \mathrm{~dB}$ using an $(1944,972)$ LDPC code compared to an ideally filtered Costas loop. For a constant phase model the gain for the DDCS circuit was around $2 \mathrm{~dB}$ for an $(1944,972)$ LDPC code and $1 \mathrm{~dB}$ for a $(648,324)$ code. Note that there is a difference in loop-SNR of around $6 \mathrm{~dB}$ between a loop that 


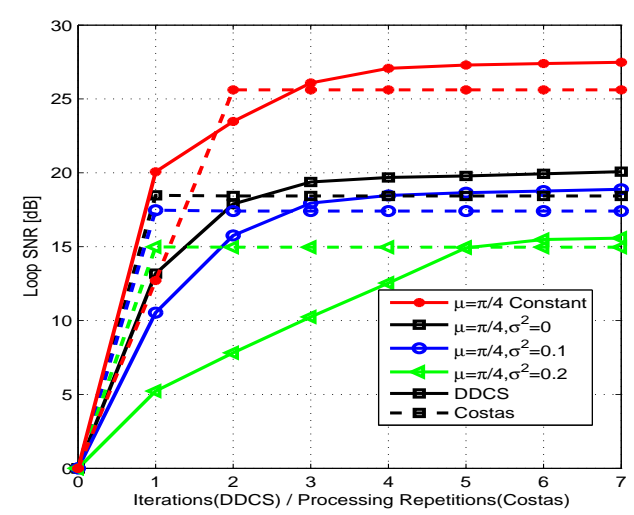

Fig. 3. Comparison of Loop-SNR for a BPSK system with $\theta_{c}=$ Uniform $[-\pi / 4, \pi / 4]$ and $E_{b} / N_{o}=1.50 \mathrm{~dB}$ using the DDCS circuit and a Costas loop and two different carrier phase models. The case labeled $\left[\mu=\pi / 4, \sigma^{2}=0\right]$ corresponds to a circuit that can track random walks but is used to track a constant phase offset. On the other hand $[\mu=\pi / 4$, Constant $]$ uses a circuit that can only track constant offsets. For this case, the $2 \mathrm{~dB}$ gain in loop-SNR for the longest LDPC code is close to the expected theoretical gain.

can only track constant phase offsets and one that can track a random-walk but is used to track constant phases.

When tracking a constant phase, on every iteration, all $n$ estimates from the phase-detection circuit are used to derive a single phase estimated value $\hat{\theta}_{c}$. When the phase behaves as in (10), in every iteration, the different $n$ estimates correspond to the $n$ components of the vector $\overrightarrow{\hat{\theta}}_{c}$. The difference in the amount of information used to compute $\hat{\theta}_{c}$ in each case $(n$ estimates per iteration for the constant-phase case and one estimate per vector component per iteration for the Wiener case) causes the $6 \mathrm{~dB}$ difference in loop-SNR performance. Furthermore, when a constant phase offset is being tracked, only the steady-state estimates from the PLL could be used to estimate $\hat{\theta}_{c}$.

For the case of a traditional $\mathrm{CL}$, the carrier recovery circuit is independent of the decoder decisions and therefore uses the same channel information every time it runs. This implies that for the CL case, the horizontal axis of Fig. 3 in fact represents the number of times that each block (of size $n$ ) is processed by the loop. For example, after 3 loop updates, the DDCS circuit has processed $S=3 n$ different symbols. After every loop update the received symbol is de-rotated and a "new" observation vector is fed to the decoder. On the other hand the CL overprocesses the same $S=n$ set of symbols every time it runs.

\section{B. Normalized Complex Conjugate (NCC) Feedback}

An alternative technique to remove the modulation information present in (2) normalizes the complex conjugate of the estimated symbol output from the LDPC decoder to unit amplitude,

$$
U(t)=Y_{R}(t) \hat{M}^{*}(t) /\|\hat{M}(t)\| .
$$

The noise affecting the loop has also been affected by the normalization since the estimation vectors used for feedback are now constrained to unit amplitude. Simulation results shown in Section IV, show a small gain in FER performance is obtained when using the NCC metric, in particular for cases that exhibit a large carrier phase offsets. Unless noted otherwise, all further plots shown in this work use NCC as a feedback metric.

\section{Iterative Processing FOR Phase Tracking}

Let $\psi$ be the rotational invariance angle of a constellation used for symbol modulation. For the constellations considered in this work we have $\psi_{\text {MPSK }}=2 \pi / M$, and $\psi_{16 \mathrm{QAM}}=\pi / 2$. Let $\gamma_{c}$ be the maximum carrier phase rotation that the system can handle without severely degrading the FER performance. While it is always desirable to increase $\gamma_{c}$ when possible, the overall phase tracking ability of the DDCS system is not limited by the value of $\gamma_{c}$. Depending on the type of modulation used, techniques that use the DDCS algorithm can be implemented to track all possible values of $\theta_{c} \in[-\pi, \pi]$.

One method for tracking offsets in $[-\pi, \pi]$ of a BPSK modulation was introduced in [1]. For this case, $\gamma_{c(B P S K)} \approx \pi / 2$, therefore the possible phase spectrum can be divided into two intervals. The decoder begins by measuring the average power across a single codeblock of the signals $v_{I_{k}}$ and $v_{Q_{k}}$. If the sine component $\left(v_{Q_{k}}\right)$ has average power greater than the cosine component $\left(v_{I_{k}}\right)$, then these two components are swapped. This procedure may leave (or induce) a remaining error of $\pi$ radians. To resolve this ambiguity we run a single PLL pass followed by several (up to 4) LDPC iterations. The orientation that produces the maximum number of satisfied odd-degree check equations is selected and the decoding procedure is re-initialized ${ }^{1}$. Similar techniques to resolve initial phase ambiguity are proposed in [9], [10].

For higher order M-ary modulations, a search method can be implemented based on the window-search method for symbol timing recovery presented in [15], [16]. The carrier phase spectrum is divided into fractions of size $2 \gamma_{c}$. The search space will therefore consist of the intervals $\left\{\left[ \pm \gamma_{c}\right),\left[\gamma_{c}, 3 \gamma_{c}\right), \ldots,[2 \pi-\right.$ $\left.\left.2 \gamma_{c}, 2 \pi-\gamma_{c}\right)\right\}$. If larger intervals are used, the DDCS system will still acquire. However, this will come at a cost of an error rate degradation. The FER penalty (early flooring) that occurs when a window size larger than $\gamma_{c(16 Q A M)}=0.17 \pi$ is chosen appears in Fig. 4(a). The received waveform is pre-rotated to fall into each potential interval (such that the maximum offset of the pre-rotated signal is limited to $\pm \gamma_{c}$ ) before beginning the DDCS estimation process. A small number of LDPC iterations (less than 4 iterations per search interval is sufficient) can be performed for each potential interval and the one with the highest number of satisfied constraints is chosen to start the final DDCS estimation. The utility of this metric as a feedback mechanism is introduced in [16].

The iterative pilotless methods mentioned in the paragraph above incur additional complexity due to the iterative process required to bring the DDCS within pull-in range. At higher SNRs where pilot insertion loss is less significant the use of pilots is an alternative option [3]. One could use pilots to

\footnotetext{
${ }^{1}$ Even degree checks remain satisfied under a rotation of all inputs by $\pi$.
} 
provide the initial phase estimates instead of the methods in [1], [15], [16]. After the initial phase-ambiguity is resolved, the proposed DDCS system will complete the acquisition and tracking showing no BER degradation compared to ideal AWGN behavior.

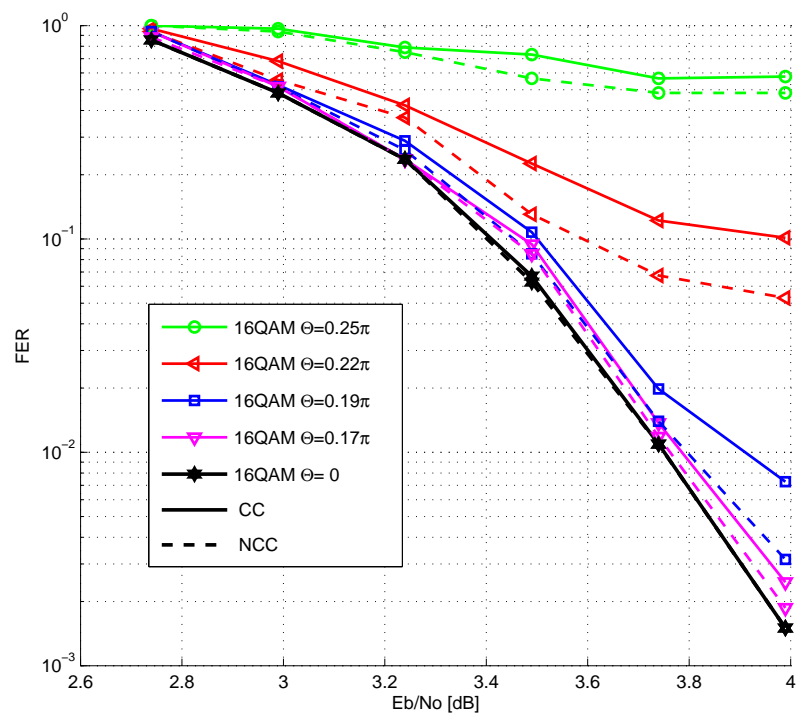

(a) $\mathrm{CC}$ vs $\mathrm{NCC}$

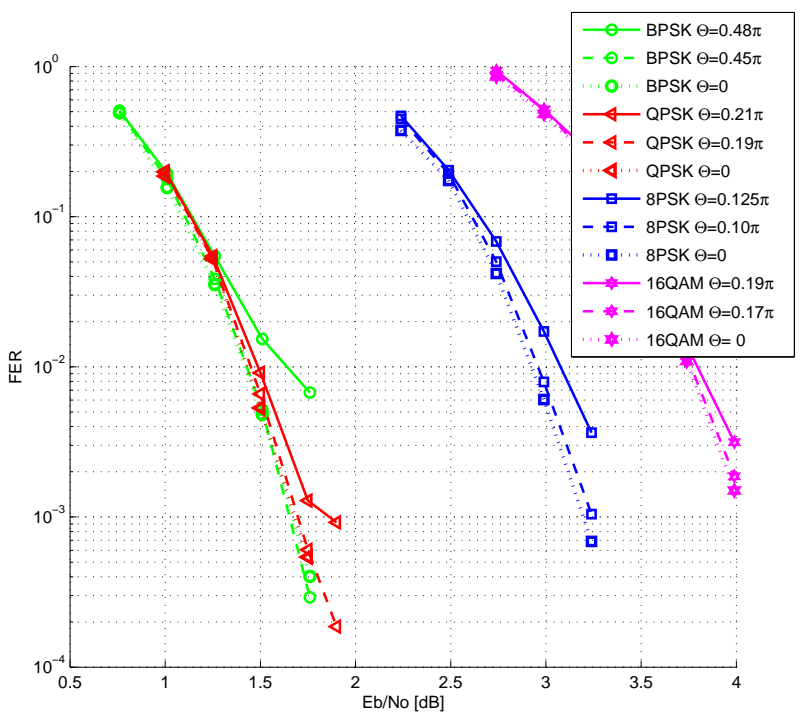

(b) FER using NCC

Fig. 4. (a) FER performance of DDCS circuit using 16-QAM and two feedback techniques: Normalized Complex Conjugate (NCC) and Complex Conjugate (CC) feedback. It is assumed that a method from Section III is used to de-rotate the incoming signal and reduce initial phase uncertainty $(0,2 \pi)$ to the cases shown in the figure. As long as the initial search space is adequate (For 16QAM, intervals are: $\pm 0.17 \pi, 0.17 \pi-0.51 \pi$ ), etc) no performance loss occurs. (b) FER performance of DDCS circuit for different modulations using a $(1944,972)$ LDPC Code and Normalized Complex Conjugate (NCC) feedback. Constant phase offset is assumed in all cases and is chosen randomly in the interval $[0,2 \pi]$. For all cases, performance is shown when no carrier offset is present and for threshold values of $\theta_{c}$

\section{Numerical Results}

\section{A. Experimental values of $\gamma_{c}$}

For the BPSK and QPSK cases,we can see in Fig. 5(b) how $\gamma_{c(B P S K)} \approx 0.3 \pi$ and $\gamma_{c(Q P S K)} \approx 0.1 \pi$ when the Wiener model in (10) is used. The figure also shows that strong random walks with $\sigma_{B P S K}^{2} \leq 0.01[\mathrm{rad}] \rightarrow$ $\sigma_{B P S K}=0.1$ can be tracked without considerable performance losses. If a constant phase offset is assumed, Fig. 5(a) shows how $\gamma_{c(B P S K)} \approx 0.45 \pi, \gamma_{c(Q P S K)} \approx 0.19 \pi$, $\gamma_{c(16 Q A M)} \approx 0.17 \pi \gamma_{c(4+4-A P S K)} \approx 0.12 \pi$ and $\gamma_{c(8 P S K)} \approx 0.10 \pi$. The APSK constellations used were obtained from [17]. In all these cases $\gamma_{c}<\frac{\psi}{2}$. It is important to emphasize once again that $\gamma_{c}$ is not the pull-in range of the carrier recovery circuit. It is the range of offsets that can be tracked while having a BER performance corresponding to "ideal" conditions where no carrier offsets are present. The DDCS system is still able to track in scenarios where the difference between the initial estimate (obtained through the use of pilots or the proposed search algorithm that uses information from constraint nodes) and the actual carrier phase is larger than $\gamma_{c}$. We can see in Fig. 4(a) the effect an early error floor when choosing search intervals that are greater than $\gamma_{c}$. From Fig. 4(a) we can also conclude that as long as the search space is adequate no performance loss occurs compared to the genie-aided performance with $\theta_{c}=0$.

\section{B. NCC vs. CC Feedback}

In Fig. 4(a) the FER performance for the two proposed methods for modulation removal is shown using a 16QAM modulation. The NCC technique outperforms the CC technique for cases of non-constant envelope constellations as the one shown. For constant envelope constellations the gap in performance between NCC and CC is reduced, although NCC seems to have a significant advantage for scenarios with a strong carrier phase offset. In Fig. 4(b), the NCC technique is used to decode different modulations using a $(1944,972)$ LDPC code and a constant phase offset. Similarly to the 16QAM case, the appropriate search intervals required to de-mitigate the initial phase ambiguity can be derived from this figure. When the Wiener model in (10) is used, the corresponding results are shown in Fig. 5(b). The loop-SNR degradation shown in Fig. 3 can be also appreciated from a FER perspective in Fig. 5(b), since the Wiener model is more sensitive to carrier errors.

\section{CONCLUSion}

We have demonstrated a means for improving the carrier synchronization function for iteratively decoded $M$-ary constellations using information derived from the decoder (decision-directed) to remove the modulation prior to the carrier tracking operation. The motivation for doing this is to overcome the penalty in noisy reference loss attributed to the large squaring loss at low SNRs that is characteristic of the traditional carrier loops such as the Costas-type loop. In contrast with the decision-directed carrier synchronization 


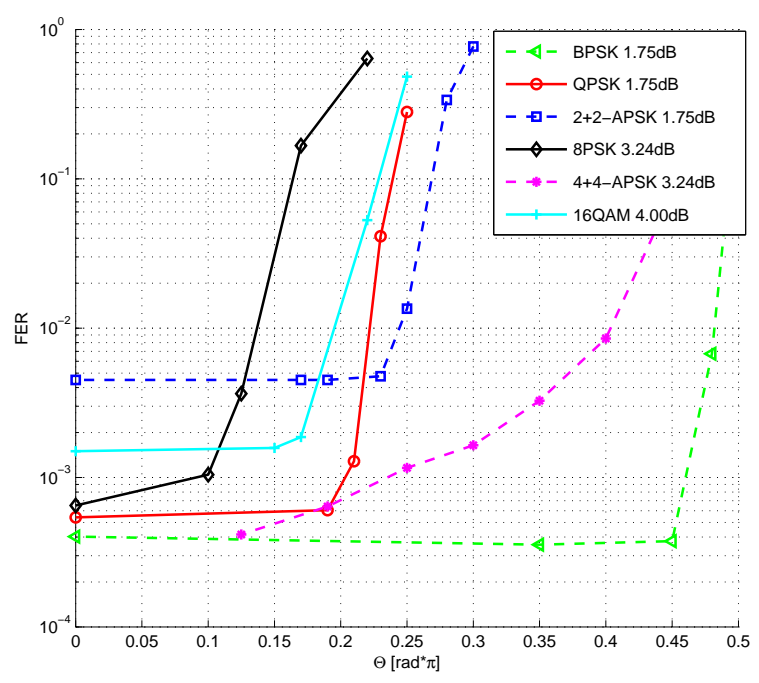

(a) Constant Phase Offset

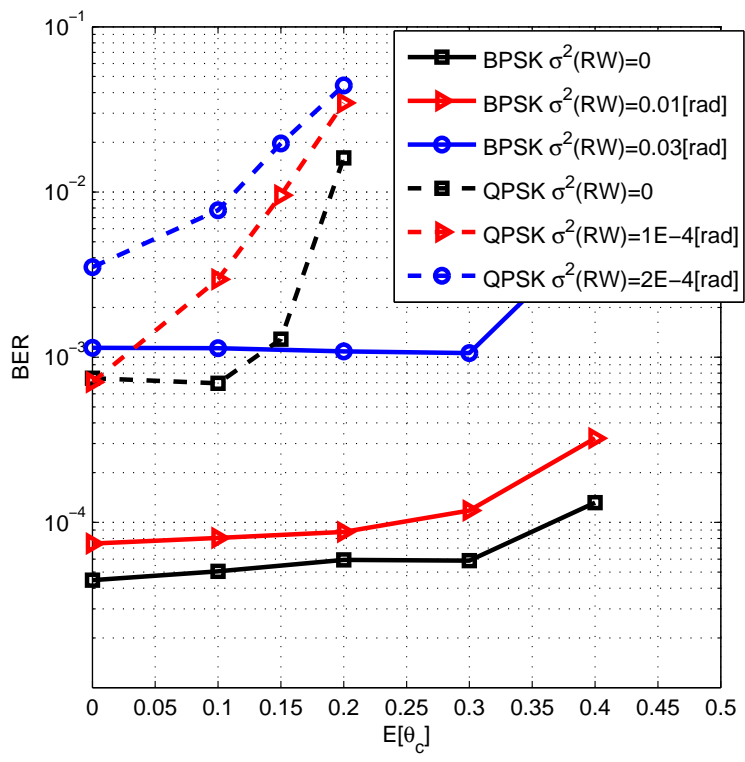

(b) Random Walk

Fig. 5. (a) FER as a function of a constant carrier phase offset $\theta_{c}$ for a fixed $E_{b} / N_{0}$. From this figure we can observe how $\gamma_{\mathrm{BPSK}} \approx 0.45 \pi, \gamma_{\mathrm{QPSK}}=$ $\gamma_{4+4-\mathrm{APSK}} \approx 0.16 \pi, \gamma_{2+2 \text {-APSK }} \approx 0.23 \pi, \gamma_{8 \mathrm{PSK}} \approx 0.1 \pi$ and $\gamma_{16-\mathrm{QAM}} \approx$ $0.17 \pi$. (b) FER performance of DDCS circuit using BPSK an a $(1944,972)$ LDPC Code. BPSK case at $E_{b} / N_{o}=1.7 \mathrm{~dB}$, QPSK case at $E_{b} / N_{o}=1.5 \mathrm{~dB}$.

loop with hard decision feedback as proposed in [14], the scheme described in this work makes use of soft-decision information and does not require estimating the decoder error probability. This occurs as a consequence of the assumption here of a fixed carrier synchronization structure, i.e., a PLL, whose design does not change with knowledge obtained from the decoder. The proposed architecture can be used in conjunction with other types of phase tracking loops in order to track residual carrier-phase errors at the decoding stage. The proposed decision-directed loop is able to track carrier offsets using an unmodified iterative decoder and a simple PLL while showing no BER degradation compared to the "ideal" AWGN performance of the LDPC code.

\section{REFERENCES}

[1] M. Simon, E.L. Vallés, C. Jones, R. Wesel, and J. Villasenor, "Information-reduced carrier synchornization of BPSK and QPSK using soft decision feedback," Proc. IEEE 44th Allerton Conf. on Comm., Control and Comput., Sep. 2006.

[2] N. Noels, V. Lottici, A. Dejonghe, H. Steendam, M. Moeneclaey, M. Luise, and L. Vandendorpe, "A theoretical framework for soft-information-based synchronization in iterative (turbo) receivers," EURASIP Journal on Wireless Communications and Networking, pp. 117-129, 2005.

[3] A. Anastasopoulos and K. Chugg, "Adaptive iterative detection for phase tracking in turbo-coded systems," IEEE Trans. on Comm., vol. 49, no. 12 , pp. 2135-2143, Dec. 2001.

[4] J. Dauwels and H.-A. Loeliger, "Phase estimation by message passing," Proc. IEEE Int. Conf. on Communications, pp. 523-527, June 2004.

[5] G. Colavolpe, A. Barbieri, and G. Caire, "Algorithms for iterative decoding in the presence of strong phase noise," IEEE J. Select. Areas Comm., vol. 23, no. 9, pp. 1748- 1757, Sept. 2005

[6] S. Howard and C. Schlegel, "Differentially-encoded turbo coded modulation with APP channel estimation," in Proc. IEEE Global Telecomm. Conf., Dec. 2003, pp. 1761-1765.

[7] R. Nuriyev and A. Anastasopoulos, "Pilot-symbol-assisted coded transmission over the block-noncoherent AWGN channel," IEEE Trans. on Comm., vol. 51, no. 6, pp. 953-963, 2003.

[8] M. Moeneclaey, N. Noels, and H. Steendam, "Performance analysis of ML-based feedback carrier phase synchronizers for coded signals," IEEE Trans. on Signal Proc., vol. 55, no. 3, pp. 1129-1136, 2007.

[9] W. Oh and K. Cheun, "Joint decoding and carrier phase recovery," IEEE Comm. Letters, vol. 5, no. 9, pp. 375-377, 2001.

[10] A. Burr and L. Zhang, "A novel carrier phase recovery method for turbocoded QPSK system," Proc. European Wireless (EW'02). Florence, Italy, pp. 917-821, Feb. 2002.

[11] V. Lottici and M. Luise, "Embedding carrier phase recovery into iterative decoding of turbo-coded linear modulations," IEEE Trans. on Comm., vol. 52, no. 4, pp. 661-669, 2004.

[12] C. Langlais and M. Helard, "Phase carrier for turbo codes over a satellite link with the help of tentative decisions," 2nd International Symposium on Turbo Codes and Related Topics. Brest, France., vol. 5, pp. 439-442, 2000.

[13] N. Noels, H. Steendam, and M. Moeneclaey, "Effectiveness study of code-aided and non-code-aided ML-based feedback phase synchronizers," Proc. IEEE Int. Conf. on Comm. (ICC), vol. 7, pp. 2946-2951, June 2006.

[14] M. Simon and V. A. Vilnrotter, "Iterative information-reduced carrier synchronization using decision feedback for low SNR applications," TDA Progress Report, vol. 42-130, Aug. 15, 1997. [Online]. Available: http://tmo.jpl.nasa.gov/progress_report/42-130/130A.pdf

[15] E.L. Vallés, C. Jones, J. Villasenor, and C. Jones, "Carrier and timing synchronization of BPSK via LDPC code feedback," Proc. IEEE Asilomar Conf. on Sig., Syst. and Comput., Nov. 2006.

[16] D. Lee, E.L. Vallés, J. Villasenor, and C. Jones, "Joint LDPC decoding and timing recovery using code constraint feedback," IEEE Comm. Letters, vol. 10, no. 3, pp. 189-191, Mar. 2006.

[17] E.L. Vallés, "Constellation design for improved iterative LDPC decoding," IEEE Aerospace Conf. Big Sky, MO., Mar. 2008. 